\title{
Steinitz classes of tamely ramified nonabelian extensions of odd prime power order
}

\author{
Alessandro Cobbe
}

July 2, 2018

\begin{abstract}
The Steinitz class of a number field extension $K / k$ is an ideal class in the ring of integers $\mathcal{O}_{k}$ of $k$, which, together with the degree $[K: k]$ of the extension determines the $\mathcal{O}_{k}$-module structure of $\mathcal{O}_{K}$. We call $\mathrm{R}_{t}(k, G)$ the classes which are Steinitz classes of a tamely ramified $G$-extension of $k$. We will say that those classes are realizable for the group $G$; it is conjectured that the set of realizable classes is always a group.

In this paper we will develop some of the ideas contained in [8] to study some $l$-groups, where $l$ is an odd prime number. In particular, together with [1] we will complete the study of realizable Steinitz classes for groups of order $l^{3}$. We will also give an alternative proof of the results of [1], based on class field theory.
\end{abstract}

\section{Introduction}

Let $K / k$ be an extension of number fields and let $\mathcal{O}_{K}$ and $\mathcal{O}_{k}$ be their rings of integers. By Theorem 1.13 in [18] we know that

$$
\mathcal{O}_{K} \cong \mathcal{O}_{k}^{[K: k]-1} \oplus I
$$

where $I$ is an ideal of $\mathcal{O}_{k}$. By Theorem 1.14 in [18] the $\mathcal{O}_{k}$-module structure of $\mathcal{O}_{K}$ is determined by $[K: k]$ and the ideal class of $I$. This class is called the Steinitz class of $K / k$ and we will indicate it by $\operatorname{st}(K / k)$. Let $k$ be a number field and $G$ a finite group, then we define:

$$
\mathrm{R}_{t}(k, G)=\{x \in \mathrm{Cl}(k): \exists K / k \text { tame, } \operatorname{Gal}(K / k) \cong G, \operatorname{st}(K / k)=x\} .
$$


In this paper we will use the notations and some techniques from [8] to study the realizable classes for some $l$-groups, where $l$ is an odd prime number.

Some of the results in this paper are parts of the author's PhD thesis [7]. For earlier results see [1, [2], 3], 44, [5], 6], 9], [10], 11], 13], 14], 15], [16], [17] [20], 21] and [22].

\section{Acknowledgements}

I am very grateful to Professor Cornelius Greither and to Professor Roberto Dvornicich for their advice and for the patience they showed, assisting me in the writing of my $\mathrm{PhD}$ thesis with a lot of suggestions. I also wish to thank the Scuola Normale Superiore of Pisa, for its role in my mathematical education and for its support during the time I was working on my $\mathrm{PhD}$ thesis.

\section{Preliminary results}

We start recalling the following two fundamental results.

Theorem 1.1. If $K / k$ is a finite tame Galois extension then

$$
\mathrm{d}(K / k)=\prod_{\mathfrak{p}} \mathfrak{p}^{\left(e_{\mathfrak{p}}-1\right) \frac{[K: k]}{e_{\mathfrak{p}}}},
$$

where $e_{\mathfrak{p}}$ is the ramification index of $\mathfrak{p}$.

Proof. This follows by Propositions 8 and 14 of chapter III of [12].

Theorem 1.2. Assume $K$ is a finite Galois extension of a number field $k$.

(a) If its Galois group either has odd order or has a noncyclic 2-Sylow subgroup then $\mathrm{d}(K / k)$ is the square of an ideal and this ideal represents the Steinitz class of the extension.

(b) If its Galois group is of even order with a cyclic 2-Sylow subgroup and $\alpha$ is any element of $k$ whose square root generates the quadratic subextension of $K / k$ then $\mathrm{d}(K / k) / \alpha$ is the square of a fractional ideal and this ideal represents the Steinitz class of the extension.

Proof. This is a corollary of Theorem I.1.1 in [9. In particular it is shown in [9] that in case (b) $K / k$ does have exactly one quadratic subextension. 
Further, considering Steinitz classes in towers of extensions, we will need the following proposition.

Proposition 1.3. Suppose $K / k_{1}$ and $k_{1} / k$ are number fields extensions. Then

$$
\operatorname{st}(K / k)=\operatorname{st}\left(k_{1} / k\right)^{\left[k_{1}: E\right]} \mathrm{N}_{k_{1} / k}\left(\operatorname{st}\left(K / k_{1}\right)\right) .
$$

Proof. This is Proposition I.1.2 in [9].

We will also use some other preliminary results.

Lemma 1.4. Let $m, n, x, y$ be integers. If $x \equiv y(\bmod m)$ and any prime $q$ dividing $n$ divides also $m$ then

$$
x^{n} \equiv y^{n} \quad(\bmod m n) .
$$

Proof. Let $n=q_{1} \ldots q_{r}$ be the prime decomposition of $n\left(q_{i}\right.$ and $q_{j}$ with $i \neq j$ are allowed to be equal). We prove by induction on $r$ that $x^{n} \equiv y^{n}$ $(\bmod m n)$. If $r=1$, then $m n=m q_{1}$ must divide $m^{q_{1}}$ and there exists $b \in \mathbb{N}$ such that

$$
x^{n}=(y+b m)^{q_{1}}=y^{q_{1}}+\sum_{i=1}^{q_{1}-1}\left(\begin{array}{c}
q_{1} \\
i
\end{array}\right)(b m)^{i} y^{q_{1}-i}+(b m)^{q_{1}} \equiv y^{n} \quad(\bmod m n) .
$$

Let us assume that the lemma is true for $r-1$ and prove it for $r$. Since $q_{r} \mid m$, as above, for some $c \in \mathbb{N}$ we have

$$
\begin{aligned}
x^{n} & =\left(y^{q_{1} \ldots q_{r-1}}+c m q_{1} \ldots q_{r-1}\right)^{q_{r}} \\
& =y^{n}+\sum_{i=1}^{q_{r}}\left(\begin{array}{c}
q_{r} \\
i
\end{array}\right)\left(c m q_{1} \ldots q_{r-1}\right)^{i} y^{q_{1} \ldots q_{r-1}\left(q_{r}-i\right)} \equiv y^{n} \quad(\bmod m n) .
\end{aligned}
$$

Definition 1.5. Let $K / k$ be a finite abelian extension of number fields. Then we define the subgroup $W(k, K)$ of the ideal class group of $k$ in the following equivalent ways (the equivalence is shown in [8], Proposition 1.10):

$$
\begin{aligned}
& W(k, K)=\left\{x \in J_{k} / P_{k}: x\right. \text { contains infinitely many primes of absolute } \\
& \text { degree } 1 \text { splitting completely in } K\} \\
& W(k, K)=\left\{x \in J_{k} / P_{k}: x \text { contains a prime splitting completely in } K\right\} \\
& W(k, K)=\mathrm{N}_{K / k}\left(J_{K}\right) \cdot P_{k} / P_{k} \text {. }
\end{aligned}
$$

In the case of cyclotomic extensions we will also use the shorter notation $W(k, m)=W\left(k, k\left(\zeta_{m}\right)\right)$ 
Lemma 1.6. If $q|n \Rightarrow q| m$ then $W(k, m)^{n} \subseteq W(k, m n)$.

Proof. Let $x \in W(k, m)$. According to Proposition 1.10 and Lemma 1.11, both from [8], $x$ contains a prime ideal $\mathfrak{p}$, prime to $m n$ and such that $\mathrm{N}_{k / \mathbb{Q}}(\mathfrak{p}) \in P_{\mathbb{Q}}^{\mathfrak{m}}$, where $\mathfrak{m}=m \cdot p_{\infty}$. Then by Lemma $1.4, \mathrm{~N}_{k / \mathbb{Q}}\left(\mathfrak{p}^{n}\right) \in P_{\mathbb{Q}}^{\mathfrak{n}}$, with $\mathfrak{n}=m n \cdot p_{\infty}$, and it follows from Lemma 1.12 of [8] that $x^{n} \in W(k, m n)$.

Definition 1.7. We will call a finite group $G$ of order $m$ good if the following properties are verified:

1. For any number field $k, \mathrm{R}_{t}(k, G)$ is a group.

2. For any tame $G$-extension $K / k$ of number fields there exists an element $\alpha_{K / k} \in k$ such that:

(a) If $G$ is of even order with a cyclic 2-Sylow subgroup, then a square root of $\alpha_{K / k}$ generates the quadratic subextension of $K / k$; if $G$ either has odd order or has a noncyclic 2-Sylow subgroup, then $\alpha_{K / k}=1$.

(b) For any prime $\mathfrak{p}$, with ramification index $e_{\mathfrak{p}}$ in $K / k$, the ideal class of

$$
\left(\mathfrak{p}^{\left(e_{\mathfrak{p}}-1\right) \frac{m}{e_{\mathfrak{p}}}-v_{\mathfrak{p}}\left(\alpha_{K / k}\right)}\right)^{\frac{1}{2}}
$$

is in $\mathrm{R}_{t}(k, G)$.

3. For any tame $G$-extension $K / k$ of number fields, for any prime ideal $\mathfrak{p}$ of $k$ and any rational prime $l$ dividing its ramification index $e_{\mathfrak{p}}$, the class of the ideal

$$
\mathfrak{p}^{(l-1) \frac{m}{e \mathfrak{p}(l)}}
$$

is in $\mathrm{R}_{t}(k, G)$ and, if 2 divides $(l-1) \frac{m}{e_{\mathfrak{p}}(l)}$, the class of

$$
\mathfrak{p}^{\frac{l-1}{2} \frac{m}{e_{\mathfrak{p}}(l)}}
$$

is in $\mathrm{R}_{t}(k, G)$.

4. $G$ is such that for any number field $k$, for any class $x \in \mathrm{R}_{t}(k, G)$ and any integer $a$, there exists a tame $G$-extension $K$ with Steinitz class $x$ and such that every non trivial subextension of $K / k$ is ramified at some primes which are unramified in $k\left(\zeta_{a}\right) / k$. 


\section{Some l-groups}

In [1, Clément Bruche proved that if $G$ is a nonabelian group of order $l^{3}=u v$ and exponent $v$, where $l$ is an odd prime, then $\mathrm{R}_{t}(k, G)=W(k, l)^{u(l-1) / 2}$ under the hypothesis that the extension $k\left(\zeta_{v}\right) / k\left(\zeta_{l}\right)$ is unramified, thereby giving an unconditional result when $G$ has exponent $l$.

In this section we prove that $\mathrm{R}_{t}\left(k, C\left(l^{2}\right) \rtimes_{\mu} C(l)\right)=W(k, l)^{l(l-1) / 2}$, without any additional hypothesis on the number field $k$. Indeed we will consider a more general situation, studying groups of the form $G=C\left(l^{n}\right) \rtimes_{\mu} C(l)$, with $n \geq 2$, where $\mu$ sends a generator of $C(l)$ to the elevation to the $l^{n-1}+1$-th power. Together with Bruche's result this will conclude the study of realizable Steinitz classes for tame Galois extensions of degree $l^{3}$.

Lemma 2.1. Let $l$ be an odd prime. The group $G=C\left(l^{n}\right) \rtimes_{\mu} C(l)$, with $n \geq 2$ is identified by the exact sequence

$$
1 \rightarrow C\left(l^{n}\right) \rightarrow G \rightarrow C(l) \rightarrow 1
$$

if the action of $C(l)$ on $C\left(l^{n}\right)$ is given by $\mu$.

Proof. Let $G$ be the group written in the above exact sequence, let $H$ be a subgroup of $G$ isomorphic to $C\left(l^{n}\right)$ and generated by $\tau$; let $x \in G$ be such that its class modulo $H$ generates $G / H$, which is cyclic of order $l$, and such that $x \tau x^{-1}=\tau^{l^{n-1}+1}$, i.e. $x \tau=\tau^{l^{n-1}+1} x$. Then $x^{l}=\tau^{a}$ for some $a \in \mathbb{N}$. Since $G$ is of order $l^{n+1}$ and it is not cyclic, the order of $x$ must divide $l^{n}$ and so

$$
\tau^{a l^{n-1}}=x^{l^{n}}=1,
$$

i.e. $l$ divides $a$ and there exists $b \in \mathbb{N}$ such that $a=b l$. By induction we prove that, for $m \geq 1$,

$$
\left(\tau^{-b} x\right)^{m}=\tau^{-b m-b l^{n-1}(m-1) m / 2} x^{m} .
$$

This is obvious for $m=1$; we have to prove the inductive step:

$$
\begin{aligned}
\left(\tau^{-b} x\right)^{m} & =\tau^{-b(m-1)-b l^{n-1}(m-2)(m-1) / 2} x^{m-1} \tau^{-b} x \\
& =\tau^{-b(m-1)-b l^{n-1}(m-2)(m-1) / 2} x^{m-1} \tau^{-b} x^{-(m-1)} x^{m} \\
& =\tau^{-b(m-1)-b l^{n-1}(m-2)(m-1) / 2} \tau^{-b\left(1+l^{n-1}\right)^{m-1}} x^{m} \\
& =\tau^{-b(m-1)-b l^{n-1}(m-2)(m-1) / 2-b-b(m-1) l^{n-1}} x^{m} \\
& =\tau^{-b m-b l^{n-1}(m-1) m / 2} x^{m} .
\end{aligned}
$$

Then calling $\sigma=\tau^{-b} x$, we obtain that

$$
\sigma^{l}=\left(\tau^{-b} x\right)^{l}=\tau^{-b l} x^{l}=\tau^{-a+a}=1 .
$$


Further

$$
\sigma \tau \sigma^{-1}=\tau^{-b} x \tau x^{-1} \tau^{b}=\tau^{-b} \tau^{l^{n-1}+1} \tau^{b}=\tau^{l^{n-1}+1}
$$

and $\sigma, \tau$ are generators of $G$. Thus $G$ must be a quotient of the group

$$
\left\langle\sigma, \tau: \sigma^{l}=\tau^{l^{n}}=1, \sigma \tau \sigma^{-1}=\tau^{l^{n-1}+1}\right\rangle .
$$

But this group has the same order of $G$ and thus they must be equal.

It follows that we can use Proposition 2.13 of [8] to study $\mathrm{R}_{t}\left(k, C\left(l^{n}\right) \rtimes_{\mu}\right.$ $C(l)$ ), for any number field $k$.

For any $\tau \in H$ we define $E_{k, \mu, \tau}$ as the fixed field in $k\left(\zeta_{o(\tau)}\right)$ of

$$
G_{k, \mu, \tau}=\left\{g \in \operatorname{Gal}\left(k\left(\zeta_{o(\tau)}\right) / k\right): \exists g_{1} \in \mathcal{G}, \mu\left(g_{1}\right)(\tau)=\tau^{\nu_{k, \tau}\left(g_{2}\right)}\right\}
$$

where $g_{2}\left(\zeta_{o(\tau)}\right)=\zeta_{o(\tau)}^{\nu_{k, \tau}\left(g_{2}\right)}$ for any $g_{2} \in \operatorname{Gal}\left(k\left(\zeta_{o(\tau)}\right) / k\right)$.

Lemma 2.2. Let $\tau$ be a generator of $C\left(l^{n}\right)$ in $C\left(l^{n}\right) \rtimes_{\mu} C(l)$. Then $E_{k, \mu, \tau}=$ $k\left(\zeta_{l^{n-1}}\right)$.

Proof. By definition $E_{k, \mu, \tau}$ is the fixed field in $k\left(\zeta_{l^{n}}\right)$ of

$$
\begin{aligned}
G_{k, \mu, \tau} & =\left\{g \in \operatorname{Gal}\left(k\left(\zeta_{l^{n}}\right) / k\right): \exists g_{1} \in C(l) \mu\left(g_{1}\right)(\tau)=\tau^{\nu_{k, \tau}(g)}\right\} \\
& =\left\{g \in \operatorname{Gal}\left(k\left(\zeta_{l^{n}}\right) / k\right): \exists a \in \mathbb{N} \tau^{a l^{n-1}+1}=\tau^{\nu_{k, \tau}(g)}\right\} \\
& =\left\{g \in \operatorname{Gal}\left(k\left(\zeta_{l^{n}}\right) / k\right): \nu_{k, \tau}(g) \equiv 1 \quad\left(\bmod l^{n-1}\right)\right\} \\
& =\left\{g \in \operatorname{Gal}\left(k\left(\zeta_{l^{n}}\right) / k\right): g\left(\zeta_{l^{n-1}}\right)=\zeta_{l^{n-1}}\right\}=\operatorname{Gal}\left(k\left(\zeta_{l^{n}}\right) / k\left(\zeta_{l^{n-1}}\right)\right) .
\end{aligned}
$$

Hence $E_{k, \mu, \tau}=k\left(\zeta_{l^{n-1}}\right)$.

Lemma 2.3. We have

$$
\mathrm{R}_{t}\left(k, C\left(l^{n}\right) \rtimes_{\mu} C(l)\right) \supseteq W\left(k, l^{n-1}\right)^{\frac{l-1}{2} l} .
$$

Further, for any $x \in W\left(k, l^{n-1}\right)$ and any positive integer a, there exists a tame $G$-extension of $k$ with Steinitz class $x^{\frac{l-1}{2} l}$ and such that any nontrivial subextension of $K / k$ is ramified at some primes which are unramified in $k\left(\zeta_{a}\right) / k$.

Proof. By Proposition 2.13 of [8] and Lemma 2.1,

$$
\mathrm{R}_{t}\left(k, C\left(l^{n}\right) \rtimes_{\mu} C(l)\right) \supseteq \mathrm{R}_{t}(k, C(l))^{l^{n}} \cdot W\left(k, E_{k, \mu, \tau}\right)^{\frac{l-1}{2} l},
$$

where $\tau$ is a generator of $C\left(l^{n}\right)$. We easily conclude since $1 \in \mathrm{R}_{t}(k, C(l))$ and, by Lemma $2.2, E_{k, \mu, \tau}=k\left(\zeta_{l^{n-1}}\right)$, i.e.

$$
W\left(k, E_{k, \mu, \tau}\right)=W\left(k, l^{n-1}\right) .
$$

The second part of the lemma follows again by Proposition 2.13 of [8]. 
To prove the opposite inclusion we need some lemmas.

Lemma 2.4. Let $\tau$ be a generator of $C\left(l^{n}\right)$ in $C\left(l^{n}\right) \rtimes_{\mu} C(l)$ and $0<c<n$ be an integer, then

$$
G_{k, \mu, \tau^{c}}^{l^{c}} \subseteq G_{k, \mu, \tau}
$$

Proof. For any positive integer $a$ we define

$$
\hat{\mu}_{\tau^{a}}: \mathcal{G} \rightarrow\left(\mathbb{Z} / o\left(\tau^{a}\right) \mathbb{Z}\right)^{*}
$$

by $\tau^{a \hat{\mu}_{\tau}\left(g_{1}\right)}=\mu\left(g_{1}\right)\left(\tau^{a}\right)$ for all $g_{1} \in \mathcal{G}$. By definition, if $g \in G_{k, \mu, \tau^{c}}$, then there exists $g_{1} \in \mathcal{G}$ such that

$$
\tau^{l^{c} \nu_{k, \tau^{c}}(g)}=\mu\left(g_{1}\right)\left(\tau^{l^{c}}\right)=\tau^{l^{c} \hat{\mu}_{\tau^{c}}\left(g_{1}\right)} .
$$

We also observe that

$$
\zeta_{l^{n-c}}^{\nu_{k, \tau}(g)}=\zeta_{l^{n}}^{l^{c} \nu_{k, \tau}(g)}=g\left(\zeta_{l^{n}}\right)^{l^{c}}=g\left(\zeta_{l^{n-c}}\right)=\zeta_{l^{n-c}}^{\nu_{k, l^{c}}(g)}
$$

and that

$$
\tau^{l^{c} \hat{\mu}_{\tau^{c}}\left(g_{1}\right)}=\mu\left(g_{1}\right)\left(\tau^{l^{c}}\right)=\mu\left(g_{1}\right)(\tau)^{l^{c}}=\tau^{l^{c} \hat{\mu}_{\tau}\left(g_{1}\right)} .
$$

From the above equalities we deduce that

$$
\nu_{k, \tau}(g) \equiv \nu_{k, \tau^{l c}}(g) \equiv \hat{\mu}_{\tau^{l^{c}}}\left(g_{1}\right) \equiv \hat{\mu}_{\tau}\left(g_{1}\right) \quad\left(\bmod l^{n-c}\right)
$$

and therefore by Lemma 1.4 we obtain that

$$
\nu_{k, \tau}\left(g^{l^{c}}\right) \equiv \hat{\mu}_{\tau}\left(g_{1}^{l^{c}}\right) \quad\left(\bmod l^{n}\right)
$$

We conclude that

$$
\tau^{\nu_{k, \tau}\left(g^{l^{c}}\right)}=\tau^{\hat{\mu}_{\tau}\left(g_{1}^{l^{c}}\right)}=\mu\left(g_{1}^{l^{c}}\right)(\tau)
$$

and hence that $g^{l^{c}} \in G_{k, \mu, \tau}$.

Lemma 2.5. Let $\tau$ be a generator of $C\left(l^{n}\right)$ in $C\left(l^{n}\right) \rtimes_{\mu} C(l)$ and $0<c<n$ be an integer, then

$$
W\left(k, E_{k, \mu, \tau^{l^{c}}}\right)^{l^{c}} \subseteq W\left(k, l^{n-1}\right) .
$$

Proof. Let $x$ be a class in $W\left(k, E_{k, \mu, \tau^{c}}\right)$. By Proposition 1.10 in [8] there exists a prime $\mathfrak{p}$ in the class of $x$ splitting completely in $E_{k, \mu, \tau^{c}} / k$. By Theorem IV.8.4 in [19], $\mathfrak{p} \in H_{E_{k, \mu, \tau^{c}} / k}^{\mathfrak{m}}$, where $\mathfrak{m}$ is a cycle of declaration of $E_{k, \mu, \tau^{c}} / k$. Then, by Proposition II.3.3 in [19],

$$
\left.\left(\frac{k\left(\zeta_{l^{n}}\right) / k}{\mathfrak{p}}\right)\right|_{E_{k, \mu, \tau^{c}}}=\left(\frac{E_{k, \mu, \tau^{c}} / k}{\mathfrak{p}}\right)=1 .
$$


Thus

$$
\left(\frac{k\left(\zeta_{l^{n}}\right) / k}{\mathfrak{p}}\right) \in \operatorname{Gal}\left(k\left(\zeta_{l^{n}}\right) / E_{k, \mu, \tau^{l^{c}}}\right)=G_{k, \mu, \tau^{l^{c}}}
$$

and it follows by Lemma 2.4 that

$$
\left(\frac{k\left(\zeta_{l^{n}}\right) / k}{\mathfrak{p}^{l^{c}}}\right)=\left(\frac{k\left(\zeta_{l^{n}}\right) / k}{\mathfrak{p}}\right)^{l^{c}} \in G_{k, \mu, \tau^{l^{c}}}^{l^{c}} \subseteq G_{k, \mu, \tau}=\operatorname{Gal}\left(k\left(\zeta_{l^{n}}\right) / E_{k, \mu, \tau}\right) .
$$

Then

$$
\left(\frac{E_{k, \mu, \tau} / k}{\mathfrak{p}^{l^{c}}}\right)=\left.\left(\frac{k\left(\zeta_{l^{n}}\right) / k}{\mathfrak{p}^{l^{c}}}\right)\right|_{E_{k, \mu, \tau}}=1
$$

and so the class $x^{l^{c}}$ of $\mathfrak{p}^{l^{c}}$ is in $W\left(k, E_{k, \mu, \tau}\right)$, which is equal to $W\left(k, l^{n-1}\right)$ by Lemma 2.2.

Lemma 2.6. Let $K / k$ be a tamely ramified abelian extension of number fields and let $\mathfrak{p}$ be a prime ideal in $k$ whose ramification index in $K / k$ is $e$, then $\mathrm{N}_{k / \mathbb{Q}}(\mathfrak{p}) \in P_{\mathbb{Q}}^{\mathfrak{m}}$, where $\mathfrak{m}=e \cdot p_{\infty}$. In particular, by Lemma 1.12 of [8], $\mathfrak{p} \in H_{k\left(\zeta_{e}\right) / k}^{\mathfrak{m}}$ and so its class is in $W(k, e)$.

Proof. This is Lemma I.2.1 of [9].

Lemma 2.7. Let $K / k$ be a tame $C\left(l^{n}\right) \rtimes_{\mu} C(l)$-extension of number fields and let $\mathfrak{p}$ be a ramifying prime, with ramification index $e_{\mathfrak{p}}$. Then the class of

$$
\mathfrak{p}^{\frac{e \mathfrak{p}-1}{2} \frac{l^{n+1}}{e_{\mathfrak{p}}}}
$$

and the class of

$$
\mathfrak{p}^{\frac{l-1}{2} \frac{l^{n+1}}{e_{\mathfrak{p}}}}
$$

are both in

$$
W\left(k, l^{n-1}\right)^{\frac{l-1}{2} l} .
$$

Proof. The Galois group of $K / k$ is $C\left(l^{n}\right) \rtimes_{\mu} C(l)$, i.e.

$$
G=\left\langle\sigma, \tau: \sigma^{l}=\tau^{l^{n}}=1, \sigma \tau \sigma^{-1}=\tau^{l^{n-1}+1}\right\rangle .
$$

Since the ramification is tame, the inertia group at $\mathfrak{p}$ is cyclic, generated by an element $\tau^{a} \sigma^{b}$; by induction we obtain

$$
\left(\tau^{a} \sigma^{b}\right)^{m}=\tau^{a m+a b l^{n-1}(m-1) m / 2} \sigma^{b m} .
$$

The order $e_{\mathfrak{p}}$ of $\tau^{a} \sigma^{b}$ must be a multiple of $l$, since the element $\tau^{a} \sigma^{b}$ is nontrivial and $G$ is an $l$-group. Hence, recalling that $\tau^{l^{n}}=1$, we obtain that $e_{\mathfrak{p}}$ is the smallest positive integer such that

$$
\tau^{a e_{\mathfrak{p}}} \sigma^{b e_{\mathfrak{p}}}=1 .
$$


First of all we assume that $l^{2}$ divides $e_{\mathfrak{p}}$. If $l^{\beta}$ is the exact power of $l$ dividing $a$, we obtain that $e_{\mathfrak{p}}=l^{n-\beta}$ and in particular that $\beta \leq n-2$. So we have

$$
\sigma_{*}\left(\tau^{a} \sigma^{b}\right)=\sigma \tau^{a} \sigma^{b} \sigma^{-1}=\tau^{a\left(l^{n-1}+1\right)} \sigma^{b}=\left(\tau^{a} \sigma^{b}\right)^{l^{n-1}+1}
$$

and

$$
\tau_{*}\left(\tau^{a} \sigma^{b}\right)=\tau \tau^{a} \sigma^{b} \tau^{-1}=\tau^{a-b l^{n-1}} \sigma^{b}=\left(\tau^{a} \sigma^{b}\right)^{-\tilde{a} b l^{n-1-\beta}+1},
$$

where $a \tilde{a} \equiv l^{\beta}\left(\bmod l^{n}\right)$. Hence, in particular, the inertia group is a normal subgroup of $G$. Thus we can decompose our extension in $K / k_{1}$ and $k_{1} / k$ which are both Galois and such that $\mathfrak{p}$ is totally ramified in $K / k_{1}$ and unramified in $k_{1} / k$. By Lemma 2.14 of [8] the class of $\mathfrak{p}$ is in $W\left(k, E_{k, \rho, \tau^{a} \sigma^{b}}\right)$, where the action $\rho$ is induced by the conjugation in $G$ and, in particular, it sends $\tau$ to the elevation to the $-\tilde{a} b l^{n-1-\beta}+1$-th power, as seen above, and $\sigma$ to the elevation to the $l^{n-1}+1$-th power. The group $G_{k, \rho, \tau^{a} \sigma^{b}}$ consists of those elements $g$ of $\operatorname{Gal}\left(k\left(\zeta_{l^{n-\beta}}\right) / k\right)$ such that $\nu_{k, \tau^{a} \sigma^{b}}(g)$ is congruent to a product of powers of $l^{n-1}+1$ and $-\tilde{a} b l^{n-1-\beta}+1$ modulo $l^{n-\beta}$. But all these are congruent to 1 modulo $l^{n-1-\beta}$ and thus $\left.G_{k, \rho, \tau^{a} \sigma^{b}}\right|_{k\left(\zeta_{l n-1-\beta}\right)}=\{1\}$. Hence

$$
E_{k, \rho, \tau^{a} \sigma^{b}} \supseteq k\left(\zeta_{l^{n-1-\beta}}\right) \supseteq k\left(\zeta_{\frac{e_{\mathbf{p}}}{l}}\right)
$$

i.e.

$$
W\left(k, E_{k, \rho, \tau^{a} \sigma^{b}}\right) \subseteq W\left(k, \frac{e_{\mathfrak{p}}}{l}\right) .
$$

Hence, by the assumption that $l^{2} \mid e_{\mathfrak{p}}$, the class of

$$
\mathfrak{p}^{\frac{l-1}{2} \frac{l^{n+1}}{e_{\mathfrak{p}}}}
$$

is in

$$
W\left(k, \frac{e_{\mathfrak{p}}}{l}\right)^{\frac{l-1}{2} \frac{l^{n+1}}{e_{\mathfrak{p}}}} \subseteq W\left(k, l^{n-1}\right)^{\frac{l-1}{2} l}
$$

and the same is true for

$$
\mathfrak{p}^{\frac{e_{\mathfrak{p}}-1}{2} \frac{l^{n+1}}{e_{\mathfrak{p}}}} \text {. }
$$

It remains to consider the case $e_{\mathfrak{p}}=l$. We now define $k_{1}$ as the fixed field of $\tau$ and we first assume that $\mathfrak{p}$ ramifies in $K / k_{1}$. Then its inertia group in $\operatorname{Gal}\left(K / k_{1}\right)=C\left(l^{n}\right)$ is of order $l$ and thus must be generated by $\tau^{l^{n-1}}$. Hence by Lemma 2.14 of [8] the class of $\mathfrak{p}$ is in $W\left(k, E_{k, \mu, \tau^{l n-1}}\right)$ and $\mathfrak{p}^{(l-1) \frac{l^{n+1}}{e_{\mathfrak{p}}}}$ is the square of an ideal in $W\left(k, E_{k, \mu, \tau^{n-1}}\right)^{\frac{l-1}{2} l^{n}}$, which is contained in $W\left(k, E_{k, \mu, \tau}\right)^{\frac{l-1}{2} l}$ by Lemma 2.5. Hence, by Lemma 2.2, the class of

$$
\mathfrak{p}^{\frac{l-1}{2} \frac{l^{n+1}}{e_{\mathfrak{p}}}}=\mathfrak{p}^{\frac{e_{\mathfrak{p}}-1}{2} \frac{l^{n+1}}{e_{\mathfrak{p}}}}
$$


is in

$$
W\left(k, l^{n-1}\right)^{\frac{l-1}{2} l} .
$$

Finally let us consider the case of $\mathfrak{p}$ ramified in $k_{1} / k$. By Lemma 2.6 the class of $\mathfrak{p}$ is in $W(k, l)$. Hence the class of

$$
\mathfrak{p}^{\frac{l-1}{2} \frac{l^{n+1}}{e_{\mathfrak{p}}}}=\mathfrak{p}^{\frac{e \mathfrak{p}-1}{2} \frac{l^{n+1}}{e_{\mathfrak{p}}}}
$$

is in

$$
W(k, l)^{\frac{l-1}{2} l^{n}}
$$

By Lemma 1.6

$$
W(k, l)^{\frac{l-1}{2} l^{n}} \subseteq W\left(k, l^{n-1}\right)^{\frac{l-1}{2} l^{2}} \subseteq W\left(k, l^{n-1}\right)^{\frac{l-1}{2} l} .
$$

Theorem 2.8. We have

$$
\mathrm{R}_{t}\left(k, C\left(l^{n}\right) \rtimes_{\mu} C(l)\right)=W\left(k, l^{n-1}\right)^{\frac{l-1}{2} l} .
$$

Further the group $C\left(l^{n}\right) \rtimes_{\mu} C(l)$ is good.

Proof. By Theorems 1.1 and 1.2 , by Lemma 2.3 and Lemma 2.7 it is immediate that

$$
\mathrm{R}_{t}\left(k, C\left(l^{n}\right) \rtimes_{\mu} C(l)\right)=W\left(k, l^{n-1}\right)^{\frac{l-1}{2} l} .
$$

The prove that $C\left(l^{n}\right) \rtimes_{\mu} C(l)$ is good is now straightforward using the same results.

\section{$3 \quad$ Nonabelian extensions of order $l^{3}$}

As a particular case of Theorem 2.8 we state the following proposition.

Proposition 3.1. The group $C\left(l^{2}\right) \rtimes_{\mu} C(l)$ is good and

$$
\mathrm{R}_{t}\left(k, C\left(l^{2}\right) \rtimes_{\mu} C(l)\right)=W(k, l)^{\frac{l-1}{2} l} .
$$

Up to isomorphism, the only other nonabelian group of order $l^{3}$ is

$$
G=\left\langle x, y, \sigma: x^{l}=y^{l}=\sigma^{l}=1, \sigma x=x \sigma, \sigma y=y \sigma, y x=x y \sigma\right\rangle,
$$

which is a semidirect product of the normal subgroup $\langle x, \sigma\rangle \cong C(l) \times C(l)$ and the cyclic subgroup $\langle y\rangle$ of order $l$, where the action $\mu_{1}$ is given by conjugation. Clément Bruche proved in [1] that

$$
\mathrm{R}_{t}(k, G)=W(k, l)^{\frac{l-1}{2} l^{2}} .
$$


We can give a different proof of Bruche's result, using class field theory. We will also prove that the nonabelian group of order $l^{3}$ and exponent $l$ studied by Bruche is a good group.

Lemma 3.2. Let $k$ be a number field, then

$$
\mathrm{R}_{t}(k, G) \supseteq W(k, l)^{\frac{l-1}{2} l^{2}} .
$$

Further, for any $x \in W(k, l)$ and any positive integer a, there exists a tame $G$-extension of $k$ with Steinitz class $x^{\frac{l-1}{2} l^{2}}$ and such that any nontrivial subextension of $K / k$ is ramified at some primes which are unramified in $k\left(\zeta_{a}\right) / k$.

Proof. Let $x \in W(k, l)$ and $n \in \mathbb{N} \backslash\{0\}$. By Theorem 2.19 in [8] there exists a $C(l)$-extension $k_{1}$ with Steinitz class $x^{l-1}$ and which is totally ramified at some prime ideals, which are unramified in $k\left(\zeta_{a}\right) / k$. Let $\mathfrak{p}$ be one of them.

Now we would like to use Lemma 2.10 of [8] to obtain a $C(l) \times C(l)$ extension of $K / k_{1}$ which is Galois over $k$, with $\operatorname{Gal}(K / k) \cong G$. Unfortunately this is not possible since the exact sequence

$$
1 \rightarrow C(l) \times C(l) \rightarrow \mathcal{H} \rightarrow C(l) \rightarrow 1
$$

does not identify the group $\mathcal{H}$ uniquely as the group $G$. Nevertheless, the argument of that lemma at least produces a $C(l) \times C(l)$-extension of $k_{1}$, which is Galois over $k$ and with $\operatorname{st}\left(K / k_{1}\right)=1$. Further we get that $\operatorname{Gal}(K / k)$ is nonabelian of oder $l^{3}$ (since the action of $C(l)$ on $C(l) \times C(l)$ is the given one and in particular not trivial), that $K / k_{1}$ is unramified at $\mathfrak{p}$ and that any nontrivial subextension of $K / k$ is ramified at some primes which are unramified in $k\left(\zeta_{a}\right) / k$.

We want to prove that $\operatorname{Gal}(K / k) \cong G$. To this aim, we assume that this is not the case, i.e. that $\operatorname{Gal}(K / k) \cong C\left(l^{2}\right) \rtimes_{\mu} C(l)$, and we derive a contradiction. First of all, by construction, $\operatorname{Gal}\left(K / k_{1}\right) \cong C(l) \times C(l)$ and this must be a subgroup of $\operatorname{Gal}(K / k) \cong C\left(l^{2}\right) \rtimes_{\mu} C(l)$ : the only possibility is that it is the subgroup $H$ which arises by replacing $C\left(l^{2}\right)$ (the left hand factor in the semidirect product) by its subgroup of order $l$; H happens to consist of all elements of $C\left(l^{2}\right) \rtimes_{\mu} C(l)$ having order 1 or $l$. Since the prime ideal $\mathfrak{p}$ ramifies in $k_{1} / k$ and not in $K / k_{1}$, its ramification index is $l$ and, therefore, its inertia group is contained in $H$. Hence by Galois theory we conclude that the inertia field of $\mathfrak{p}$ in $K / k$ contains $k_{1}$, i.e. that $\mathfrak{p}$ ramifies in $K / k_{1}$ and not in $k_{1} / k$. This is a contradiction, since $\mathfrak{p}$ is ramified in $k_{1} / k$.

Hence we have proved that in the above construction the extension $K / k$ has Galois group G. By Proposition 1.3.

$$
\operatorname{st}(K / k)=\operatorname{st}\left(k_{1} / k\right)^{\left[K: k_{1}\right]}=x^{\frac{l-1}{2} l^{2}} \mathrm{~N}_{k_{1} / k}\left(\operatorname{st}\left(K / k_{1}\right)\right) .
$$


To prove the opposite inclusion we need the following lemma.

Lemma 3.3. Let $K / k$ be a tame $G$-extension of number fields. The ramification index of a prime ramifying in $K / k$ is $l$ and its class is contained in $W(k, l)$.

Proof. The ramification index of a ramifying prime is equal to $l$, since the corresponding inertia group must be cyclic and any nontrivial element in $G$ is of order $l$.

Let $k_{1}$ be the subfield of $K$ fixed by the normal abelian subgroup $\langle x, \sigma\rangle$ of the Galois group $G$ of $K / k$.

If a prime $\mathfrak{p}$ ramifies in $k_{1} / k$, then its class is in $W(k, l)$ by Lemma 2.6.

If a prime $\mathfrak{p}$ ramifies in $K / k_{1}$, then it is unramified in $k_{1} / k$ (the ramification index is prime) and so its inertia group is generated by an element of the form $x^{a} \sigma^{c}$, where $a, c \in\{0,1, \ldots, l-1\}$ are not both 0 . By Lemma 2.14 of [8] the class of $\mathfrak{p}$ is in $W\left(k, E_{k, \mu_{1}, x^{a} \sigma^{c}}\right)$. For any $b \in\{0,1, \ldots, l-1\}$ we have

$$
\mu_{1}\left(y^{b}\right)\left(x^{a} \sigma^{c}\right)=y^{b} x^{a} \sigma^{c} y^{-b}=x^{a} \sigma^{c+a b}
$$

and this expression cannot be a nontrivial power of $a^{a} \sigma^{c}$. Hence, by definition, the group $G_{k, \mu_{1}, x^{a} \sigma^{c}}$ must be trivial and we conclude that $E_{k, \mu_{1}, x^{a} \sigma^{c}}=$ $k\left(\zeta_{l}\right)$. Therefore, in particular, the class of the prime ideal $\mathfrak{p}$ is contained in $W(k, l)$.

Proposition 3.4. The group $G$ is good and

$$
\mathrm{R}_{t}(k, G)=W(k, l)^{\frac{l-1}{2} l^{2}} .
$$

Proof. One inclusion is given by Lemma 3.2. The proof that $\mathrm{R}_{t}(k, G) \subseteq$ $W(k, l)^{\frac{l-1}{2} l^{2}}$ follows by Lemma 3.3 , since for any tame $G$-extension $K / k$ of number fields the Steinitz class is the class of the ideal

$$
\prod_{\mathfrak{p}: e_{\mathfrak{p}} \neq 1} \mathfrak{p}^{\frac{e_{\mathfrak{p}}-1}{2} \frac{l^{3}}{e_{\mathfrak{p}}}}=\prod_{\mathfrak{p}: e_{\mathfrak{p}} \neq 1} \mathfrak{p}^{\frac{l-1}{2} l^{2}}
$$

which is contained in $W(k, l)^{\frac{l-1}{2} l^{2}}$. Now we prove that all the properties of good groups are verified.

1. This is clear, since $W(k, l)$ is a group.

2. For any prime $\mathfrak{p}$, ramifying in a tame $G$-extension $K / k$ of number fields, by Lemma 3.3, the class of

$$
\mathfrak{p}^{\frac{e_{\mathfrak{p}}-1}{2} \frac{l^{3}}{e_{\mathfrak{p}}}}=\mathfrak{p}^{\frac{l-1}{2} l^{2}}
$$

is contained in $W(k, l)^{\frac{l-1}{2} l^{2}}$, which is equal to $\mathrm{R}_{t}(k, G)$. 
3. Immediate by Lemma 3.3 and the explicit formula for $\mathrm{R}_{t}(k, G)$.

4. This follows by Lemma 3.2 .

\section{References}

[1] C. Bruche. Classes de Steinitz d'extensions non abéliennes de degré $p^{3}$. Acta Arith., 137(2):177-191, 2009.

[2] C. Bruche and B. Sodaïgui. On realizable Galois module classes and Steinitz classes of nonabelian extensions. J. Number Theory, 128(4):954978, 2008.

[3] N. P. Byott, C. Greither, and B. Sodaïgui. Classes réalisables d'extensions non abéliennes. J. Reine Angew. Math., 601:1-27, 2006.

[4] J. E. Carter. Steinitz classes of a nonabelian extension of degree $p^{3}$. Colloq. Math., 71(2):297-303, 1996.

[5] J. E. Carter. Steinitz classes of nonabelian extensions of degree $p^{3}$. Acta Arith., 78(3):297-303, 1997.

[6] J. E. Carter and B. Sodaïgui. Classes de Steinitz d'extensions quaternioniennes généralisées de degré $4 p^{r}$. J. Lond. Math. Soc. (2), 76(2):331$344,2007$.

[7] A. Cobbe. Steinitz classes of tamely ramified Galois extensions of algebraic number fields. PhD thesis, Scuola Normale Superiore, Pisa, 2010.

[8] A. Cobbe. Steinitz classes of tamely ramified Galois extensions of algebraic number fields. arXiv:0910.5080v1, to appear in Journal of Number Theory.

[9] L. P. Endo. Steinitz classes of tamely ramified Galois extensions of algebraic number fields. $\mathrm{PhD}$ thesis, University of Illinois at UrbanaChampaign, 1975.

[10] M. Godin and B. Sodaïgui. Classes de Steinitz d'extensions à groupe de Galois $A_{4}$. J. Théor. Nombres Bordeaux, 14(1):241-248, 2002.

[11] M. Godin and B. Sodaïgui. Module structure of rings of integers in octahedral extensions. Acta Arith., 109(4):321-327, 2003. 
[12] S. Lang. Algebraic number theory. GTM 110. Springer-Verlag, New York, second edition, 1994.

[13] R. Long. Steinitz classes of cyclic extensions of degree $l^{r}$. Proc. Amer. Math. Soc., 49:297-304, 1975.

[14] R. L. Long. Steinitz classes of cyclic extensions of prime degree. J. Reine Angew. Math., 250:87-98, 1971.

[15] R. Massy and B. Sodaïgui. Classes de Steinitz et extensions quaternioniennes. Proyecciones, 16(1):1-13, 1997.

[16] L. R. McCulloh. Cyclic extensions without relative integral bases. Proc. Amer. Math. Soc., 17:1191-1194, 1966.

[17] L. R. McCulloh. Galois module structure of abelian extensions. J. Reine Angew. Math., 375/376:259-306, 1987.

[18] W. Narkiewicz. Elementary and analytic theory of algebraic numbers. Springer Monographs in Mathematics. Springer-Verlag, Berlin, third edition, 2004.

[19] J. Neukirch. Class field theory, volume 280 of Grundlehren der Mathematischen Wissenschaften [Fundamental Principles of Mathematical Sciences]. Springer-Verlag, Berlin, 1986.

[20] B. Sodaïgui. Classes de Steinitz d'extensions galoisiennes relatives de degré une puissance de 2 et problème de plongement. Illinois J. Math., 43(1):47-60, 1999.

[21] B. Sodaïgui. Relative Galois module structure and Steinitz classes of dihedral extensions of degree 8. J. Algebra, 223(1):367-378, 2000.

[22] E. Soverchia. Steinitz classes of metacyclic extensions. J. London Math. Soc. (2), 66(1):61-72, 2002. 